Proceedings of the 46th International School and Conference on the Physics of Semiconductors "Jaszowiec" 2017, Szczyrk

\title{
Commutators of Jastrow Factors and Angular Momentum Operators
}

\begin{abstract}
B. Kuśmierz* AND A. Wóss
Department of Theoretical Physics, Wrocław University of Science and Technology, Wrocław, Poland

We present detailed calculations of commutators of the Jastrow factor and certain differential operators useful in the fractional quantum Hall effect. In particular, we analyze action of the angular momentum operators projected from the Haldane sphere on an arbitrary composite fermions state. Examined $L^{+}$and $L^{-}$momentum operators and following uniformity condition had proven to be useful in the search for candidates for quantum Hall ground states among many families of polynomials including the Jack polynomials.
\end{abstract}

DOI: 10.12693/APhysPolA.132.405

PACS/topics: 73.43.-f, 71.10.Pm, 02.10.Ox

\section{Introduction}

Fractional quantum Hall effect (FQHE) is remarkable behavior of electrons in high magnetic field, intensively studied for over three decades [1-6]. Despite complexity of the general problem of interacting electrons in a flat band, form of wave functions is relatively well understood. Description of multiple fermions confined to the lowest Landau level (LLL), relevant for the description of FQHE, emergence of composite fermions (CFs), and other phenomena connected with condensation of twodimensional (2D) electrons in high magnetic field into incompressible quantum liquids, have a simple mathematical structure of antisymmetric polynomials

$$
\Phi\left(z_{1}, z_{2} \ldots\right)=D\left(z_{1}, z_{2} \ldots\right) \Psi\left(z_{1}, z_{2} \ldots\right)
$$

where $z_{i}$ 's are complex electron coordinates on a plane, $\Psi$ is fully symmetric and $D$ is a Vandermonde determinant

$$
D=\prod_{i<j}\left(z_{i}-z_{j}\right) .
$$

In particular, the famous Laughlin ground state at filling factor $\nu=1 / 3[1,3]$ has the form $\Phi_{L}=D^{3}$. The Laughlin state corresponds to a completely filled Landau level of identical and essentially noninteracting composite fermions. In general, the factorization $\Phi_{C F}=D^{2 p+1} \Psi$ (multiplication by the Jastrow factor) signifies emergence of CFs - topological bound states of electrons and $2 p$ attached vortices (or magnetic flux quanta) in a state $D \Psi[1,2]$.

Certain aspects of FQHE are simpler when examined in spherical geometry (the Haldane sphere). The Haldane sphere brings useful analytical tools, for example projection of a trial wave function on the sphere imposes analytical conditions. Such analysis regarding the Jack polynomials had been done by Haldane and Bernevig [7-9]. They used lowest weight (LW) and highest

*corresponding author; e-mail: bartosz-kusmierz@o2.pl weight (HW) conditions to determine so-called "Jack states" [7-16].

In this paper we extend their calculations and analyze more general form of trial wave functions. We give detailed calculations of commutators of the Jastrow factor and certain differential operators useful in FQHE. Paper is organized as follows: in the next section we briefly discuss construction of CFs and spherical geometry, third section is devoted to the main result - calculations of commutators. In the final section we discuss results.

\section{Composite fermions, planar and spherical geometry}

The composite fermion theory $[1,2]$ explains appearance of many $\mathrm{FQH}$ states. CFs are quasiparticles, topological bound states of an electron and an even number of quantized vortices. For a system of $N$ electrons in the perpendicular, uniform magnetic field $B$, when each of them bounds $2 p$ fluxes, then in the limit of infinite $N$, each of electron experiences an effective magnetic field smaller than $B$. Filing factor of the composite fermions $\nu^{*}$ in terms of filling factor for the electrons $\nu$ is expressed as

$$
\left(\nu^{*}\right)^{-1}=\nu^{-1}-2 p .
$$

Composite fermions theory gives trial wave functions for systems of electrons $\Psi^{F Q H E}$ at filling factor $\nu$ in terms of wave function of composite fermions $\Psi^{I Q H E}$ at filling factor $\nu^{*}$

$$
\Psi^{\mathrm{FQHE}}=P_{L L L} \Psi^{\mathrm{IQHE}} \prod_{i<j}\left(z_{i}-z_{j}\right)^{2 p},
$$

where $P_{L L L}$ is a projection into the lowest Landau level.

The Haldane sphere $[1,17,18]$ is two-dimensional sphere containing electrons. Magnetic field, perpendicular to the surface is provided by the Dirac monopole in the center of the sphere. The magnetic flux through the surface of considered sphere is quantized. Value of the radial magnetic field $B$ on the surface of the sphere of radius $r$ is given by 


$$
B=\frac{N_{\Phi} \phi_{0}}{4 \pi r^{2}},
$$

where $\phi_{0}=h c / e$ and $N_{\Phi}$ is a strength of monopole. On the surface of the Haldane sphere instead of standard $\theta$ and $\varphi$ coordinates one uses $u, v$ defined as:

$$
u=\cos (\theta / 2) e^{\mathrm{i} \varphi / 2}, \quad v=\sin (\theta / 2) e^{-\mathrm{i} \varphi / 2} .
$$

For a Hilbert space of square integrable functions over $\mathbb{C}$ on a sphere, with respect to the standard, rotation invariant measure $\mathrm{d}^{2} \Omega=\sin \theta \mathrm{d} \theta \mathrm{d} \varphi$, denote by $H_{S}$ subspace of homogeneous polynomials in $u, v[8]$. Set of functions $\left\{e_{Q, m}\right\}_{Q, m}$ provide an orthonormal basis in $H_{S}$ :

$$
e_{N_{\Phi}, m}=\sqrt{\frac{2 N_{\Phi}+1}{4 \pi}\left(\begin{array}{c}
2 Q \\
N_{\Phi}+m
\end{array}\right)} u^{N_{\Phi}+m} v^{N_{\Phi}-m},
$$

where $m \in\left\{-N_{\Phi},-N_{\Phi}+1, \ldots, N_{\Phi}\right\}$. Transition from the space $H_{S}$ to the space of the wave functions in the LLL is established by linear mapping $\Gamma$ :

$$
\Gamma\left(\sum_{i=0}^{2 Q N_{\Phi}} c_{i} u^{2 N_{\Phi}-i} v^{i}\right)=\sum_{i=1}^{2 N_{\Phi}} c_{i} z^{k} .
$$

One writes angular momentum operators on the Haldane sphere

$$
\begin{aligned}
L^{X} & =\frac{1}{2}\left(v \partial_{u}+u \partial_{v}\right), \\
L^{Y} & =\frac{\mathrm{i}}{2}\left(v \partial_{u}-u \partial_{v}\right), \\
L^{Z} & =\frac{1}{2}\left(u \partial_{u}-v \partial_{v}\right) .
\end{aligned}
$$

Then

$$
\begin{aligned}
& L^{+}=L^{X}+\mathrm{i} L^{Y}=-u \partial_{v}, \\
& L^{-}=L^{X}-\mathrm{i} L^{Y}=-v \partial_{u} .
\end{aligned}
$$

By stereographic projection, operators can be applied to functions on the plane. Then operators take form

$$
\begin{aligned}
L^{+} & =E_{0}, \\
L^{-} & =N_{\Phi} Z-E_{2}, \\
L^{Z} & =\frac{1}{2} N N_{\Phi}-E_{1},
\end{aligned}
$$

where

$$
\begin{aligned}
& E_{n}=\sum_{i=1}^{N} z_{i}^{n} \partial_{i}, \\
& Z=\sum_{i=1}^{N} z_{i} .
\end{aligned}
$$

Papers [7-9] give necessary conditions for both partition and real parameter of the Jack polynomial to be a candidate for FQH ground state wave function. Bernevig and Haldane required a Jack wave function $\Psi$, to be annihilated by raising and lowering operators on the sphere $L^{+} \Psi=0, L^{-} \Psi=0$ (highest weight - HW and lowest weight - LW conditions, respectively).

\section{Results}

We calculate commutators of differential operator $E_{n}$ and the Jastrow factor $D^{m}$ (for details see Appendix A).
The Jastrow factor is to be understood as operator of multiplication by the Jastrow factor: $D^{m}: \Psi \rightarrow D^{m} \Psi$. We obtain

$$
\begin{aligned}
& {\left[E_{n}, D^{m}\right]=m D^{m} \sum_{i, j: i \neq j} \frac{z_{i}^{n}}{z_{i}-z_{j}}=} \\
& m D^{m} \sum_{i<j} \frac{z_{i}^{n}-z_{j}^{n}}{z_{i}-z_{j}} .
\end{aligned}
$$

For $n=0,1,2$ :

$$
\begin{aligned}
& {\left[E_{0}, D^{m}\right]=0,} \\
& {\left[E_{1}, D^{m}\right]=m D^{m} \frac{1}{2} N(N-1),} \\
& {\left[E_{2}, D^{m}\right]=m D^{m}(N-1) Z .}
\end{aligned}
$$

Consider action of $L^{+}, L^{-}$operators on wave function of electrons for composite fermions in a state $\Psi$. Then electrons are described by function $P_{L L L}\left(D^{m} \Psi\right)$. We analyze case of function $D^{m} \Psi$ being in $L L L$. Then one can skip projection operator and obtain

$$
\begin{aligned}
& L^{+}\left(D^{m} \Psi\right)=E_{0}\left(D^{m} \Psi\right)=D^{m} E_{0} \Psi=D^{m} L^{+} \Psi \\
& L^{-}\left(D^{m} \Psi\right)=D^{m}\left(\left(\left(N_{\Phi}-m(N-1)\right) Z-E_{2}\right) \Psi\right) .
\end{aligned}
$$

\subsection{Examples}

Consider polynomials over two variables: $x, y$, then $N=2$ and $L^{+}=\partial_{x}+\partial_{y}$. Polynomial $D^{3}+2 D=(x-$ $y)^{3}+2(x-y)$ although not homogeneous is an eigenvector of $L^{+}$with eigenvalue 0 (thus satisfies HW condition)

$$
L^{+}\left(D^{3}+D\right)=\left(L^{+} D^{3}\right)+L^{+} D=0 .
$$

For any $m, D^{m}\left(D^{3}+2 D\right)$ also satisfies HW condition

$$
L^{+} D^{m}\left(D^{3}+2 D\right)=D^{m} L^{+}\left(D^{3}+2 D\right)=D^{m} \cdot 0=0 .
$$

For $N=2$, variables $x, y$, magnetic monopole inside sphere $N_{\Phi}=3$ one writes $L^{-}=3(x+y)-x^{2} \partial_{x}-y^{2} \partial_{y}$. Polynomial $x^{3} y^{3}$ satisfies LW condition

$$
\begin{aligned}
& \left(3(x+y)-x^{2} \partial_{x}-y^{2} \partial_{y}\right) x^{3} y^{3}=3 x^{4} y^{3}+3 x^{3} y^{4} \\
& -3 x^{4} y^{3}-3 x^{3} y^{4}=0 .
\end{aligned}
$$

Function $(x-y) x^{3} y^{3}=x^{4} y^{3}-x^{3} y^{4}$ satisfy HW condition on a sphere with monopole $3+1 \cdot(2-1)=4$ since

$$
\begin{aligned}
& \left(4(x+y)-x^{2} \partial_{x}-y^{2} \partial_{y}\right) x^{4} y^{3}-x^{3} y^{4}=4 x^{5} y^{3} \\
& \quad-4 x^{3} y^{5}-\left(4 x^{5} y^{3}-3 x^{4} y^{4}+3 x^{4} y^{4}-4 x^{3} y^{5}\right)=0 .
\end{aligned}
$$

Consider any bosonic Jack state described by a Jack polynomial $J_{\lambda}^{\alpha}$ where $\alpha$ is a real number and $\lambda$ is a partition (non-increasing sequence of nonnegative integers) [19-25]. Calculations reveals that as long as bosonic Jack satisfies HW and LW conditions on a sphere with monopole $N_{\Phi}$, then corresponding fermionic Jack $D J_{\lambda}^{\alpha}$ satisfies HW and LW conditions on a sphere with monopole $N_{\Phi}+N-1$.

\section{Summary}

Calculations reveal that as long as $\Psi$ is an eigenvector of $L^{+}$with eigenvalue $e_{\Psi}^{+}$, then $D^{m} \Psi$ is en eigenvector of 
$L^{+}$with the same eigenvalue. This result is independent of the strength of a monopole of the sphere. In particularity $\Psi$ satisfy HW condition if and only if $D^{m} \Psi$ satisfies HW condition. In the case of $L^{-}$situation is similar, but not exactly the same. For $\Psi$ being eigenvector of $L^{-}$on a sphere with magnetic monopole $N_{\Phi}$, function $D^{m} \Psi$ is eigenvector of $L^{-}$on a sphere with magnetic monopole $N_{\Phi}+m(N-1)$. Thus LW condition is satisfied by $D^{m} \Psi$ on a sphere with adequately higher magnetic monopole, when LW condition is satisfied by $\Psi$ on a sphere with monopole $N_{\Phi}$.

\section{Appendix A: Calculations of comutators}

Consider the operator $\left(z_{i}^{n} \partial_{i}\right) D^{m}$ applied to the function $\Psi$. Then

$$
\begin{aligned}
& \left(z_{i}^{n} \partial_{i}\right) D^{m} \Psi=\left(z_{i}^{n} \partial_{i}\right)\left(D_{i}^{m} \cdot\left(z_{1}-z_{i}\right)^{m} \ldots\right. \\
& \left.\quad\left(z_{i-1}-z_{i}\right)^{m}\left(z_{i}-z_{i+1}\right)^{m} \ldots\left(z_{i}-z_{N}\right)^{m} \cdot \Psi\right),
\end{aligned}
$$

where $D_{i}$ is a product of all of the elements of $D$ with no variable $z_{i}$ in the brackets

$$
D_{i}=\prod_{j, k: j<k ; j, k \neq i}\left(z_{j}-z_{k}\right) .
$$

One writes

$$
\begin{aligned}
& \left(z_{i}^{n} \partial_{i}\right) D^{m} \Psi= \\
& \quad D_{i}{ }^{m}\left(\sum_{j: i<j}\left(z_{1}-z_{i}\right)^{m} \ldots\left(\left(z_{i}^{n} \partial_{i}\right)\left(z_{i}-z_{j}\right)^{m}\right) \ldots\right. \\
& \left(z_{i}-z_{N}\right)^{m} \cdot \Psi+\sum_{j: i>j}\left(z_{1}-z_{i}\right)^{m} \ldots \\
& \left(\left(z_{i}^{n} \partial_{i}\right)\left(z_{j}-z_{i}\right)^{m}\right) \ldots\left(z_{i}-z_{N}\right)^{m} \cdot \Psi \\
& \left.+\left(z_{1}-z_{i}\right)^{m} \ldots\left(z_{i}-z_{N}\right)^{m} \cdot\left(z_{i}^{n} \partial_{i}\right) \Psi\right)= \\
& \quad D^{m} \cdot \sum_{j: i<j} m \frac{z_{i}^{n}}{z_{i}-z_{j}} \cdot \Psi+D^{m} \cdot \sum_{j: i>j} m \frac{\left(-z_{i}^{n}\right)}{z_{j}-z_{i}} \cdot \Psi \\
& +D^{m} \cdot\left(z_{i}^{n} \partial_{i}\right) \Psi= \\
& \quad\left(m \sum_{j: i \neq j} \frac{z_{i}^{n}}{z_{i}-z_{j}} \cdot \Psi+\left(z_{i}^{n} \partial_{i}\right) \Psi\right)
\end{aligned}
$$

Thus

$$
\left[z_{i}^{n} \partial_{i}, D^{m}\right]=m D^{m} \sum_{j: i \neq j} \frac{z_{i}^{n}}{z_{i}-z_{j}} .
$$

What follows

$$
\begin{aligned}
& {\left[E_{n}, D^{m}\right]=m D^{m} \sum_{i, j: i \neq j} \frac{z_{i}^{n}}{z_{i}-z_{j}}=} \\
& m D^{m} \sum_{i<j} \frac{z_{i}^{n}-z_{j}^{n}}{z_{i}-z_{j}} .
\end{aligned}
$$

\section{Acknowledgments}

We acknowledge financial support from the Polish NCN Grant No. 2014/14/A/ST3/00654.

\section{References}

[1] J.K. Jain, Composite Fermions, Pennsylvania State University, 2012.

[2] J.K. Jain, Phys. Rev Lett. 63, 199 (1989).

[3] R. Laughlin, Phys. Rev. Lett. 50, 1395 (1983).

[4] D.C. Tsui, H.L. Störmer, A.C. Gossard, Phys. Rev. Lett. 48, 1559 (1982).

[5] G. Moore, N. Read, Nucl. Phys. B 360, 362 (1991).

[6] N. Read, E. Rezayi, Phys. Rev. B 59, 8084 (1999).

[7] B.A. Bernevig, F.D.M. Haldane, Phys. Rev. B 77 , 184502 (2008).

[8] B.A. Bernevig, F.D.M. Haldane, Phys. Rev. Lett. 100, 246802 (2008).

[9] B.A. Bernevig, F.D.M. Haldane, Phys. Rev. Lett. 102, 066802 (2009).

[10] A. Bernevig, N. Regnault, Phys. Rev. Lett. 103, 206801 (2009).

[11] R. Thomale, B. Estienne, N. Regnault, A. Bernevig, Phys. Rev. B 84, 045127 (2011).

[12] W. Baratta, P.J. Forrester, Nucl. Phys. B 843, 362 (2011).

[13] B. Kuśmierz, Y.-H. Wu, A. Wójs, Acta Phys. Pol. A 126, 1134 (2014).

[14] B. Kuśmierz, Y.-H. Wu, A. Wójs, Acta Phys. Pol. A 129, A-73 (2016).

[15] B. Kuśmierz, A. Wójs, Acta Phys. Pol. A 130, 1183 (2016).

[16] B. Kuśmierz, A. Wójs, Acta Phys. Pol. A 130, 607 (2016).

[17] F.D.M. Haldane, Phys. Rev. Lett. 51, 605 (1983).

[18] G. Fano, F. Ortolani, E. Colombo, Phys. Rev. B 34 2670 (1986).

[19] I.G. Macdonald, Symmetric Functions and Hall Polynomials, Oxford University Press, New York 1995.

[20] I.G. Macdonald, "A New Class of Symmetric Functions", in: Actes 20 Séminaire Lotharingien, 372/S20, Publ. I.R.M.A., Strasbourg 1988, p. 131.

[21] S. Kerov, Asymptotic Representation Theory of the Symmetric Group and Its Applications in Analysis, American Mathematical Society, USA, 2003.

[22] R.P. Stanley, Adv. Math. 77, 76 (1988).

[23] F K. Sogo, J. Math. Phys. 35, 22822296 (1994).

[24] A. Hora, N. Obata, Quantum Probability and Spectral Analysis of Graphs, Springer-Verlag, Berlin 2007.

[25] L. Lapointe, A. Lascoux, J. Morse, Electr. J. Combin. 7, N1 (2000). 